\section{Hormônio da Parativeóide (1-34) no Tratamento da Osteoporose}

\begin{abstract}
RESUMO
O hormônio da paratireóide (PTH) é produzido nas glândulas paratireóides e atua no tecido ósseo e rins, controlando a concentração sérica do cálcio e fósforo. Há muito tempo, observou-se que doses intermitentes de PTH poderiam agir anabolicamente no tecido ósseo. Vários estudos clínicos demonstram que o PTH recombinante humano (1-34) aumenta a densidade mineral óssea, promove ganho de tecido ósseo com melhora da conectividade trabecular e reduz o risco de fraturas vertebrais e extra-vertebrais em mulheres com osteoporose da pósmenopausa, mulheres com osteoporose corticóide-induzida e homens com osteoporose idiopática. A redução do risco de fraturas promovida pelo PTH (1-34) persiste após a suspensão da terapêutica, indicando seu efeito prolongado no tecido ósseo. Revisamos os dados existentes na literatura sobre o efeito anabólico do PTH (1-34) no tecido ósseo e seu uso clínico no tratamento da osteoporose. (Arq Bras Endocrinol Metab 2003;47/3:285-291)
\end{abstract}

Descritores: PTH (1-34)rh; Osteoporose; Fratura óssea; Terapêutica para osteoporose; Neo-formação óssea

\begin{abstract}
Parathyroid Hormone (1-34 PTH) for the Treatment of Osteoporosis. Parathyroid glands produce the parathyroid hormone (PTH) that acts on bone and kidneys controlling calcium and phosphorus serum concentration. It has long been known that PTH can act as an anabolic hormone if it is given by small and intermittent doses. Recently, there are data showing that PTH (1-34) increases bone mineral density, increases bone mass, improves trabecular connectivity, and reduces the risk of vertebral, non-vertebral and hip fractures in women with postmenopausal or corticoid-induced osteoporosis and in men with idiopathic osteoporosis. The effect of PTH (1-34) is sustained along the time, even when PTH (134) is withdrawn. In this article, we looked over the literature data about PTH (1-34) anabolic action in the bone and its use to treat osteoporosis. (Arq Bras Endocrinol Metab 2003;47/3:285-291)
\end{abstract}

Keywords: rhPTH (1-34); Osteoporosis; Bone fracture; Osteoporosis treatment; Bone neo-formation

O HORMÔNIO DA PARATIREÓIDE (PTH) é um hormônio de 84 aminoácidos (1), cuja fração biologicamente ativa corresponde à seqüência dos aminoácidos 1-34. O PTH liga-se a seu receptor específico no rim e osso pela fração dos aminoácidos 1-34. O receptor do PTH é denominado PTH/parathyroid hormone-related protein (PTH/PTHrP) receptor, pois pode ser ativado tanto pelo PTH quanto por fragmentos do PTHrp contendo os aminoácidos 1-34 (2). A ligação do PTH a seu receptor ativa a adenilciclase e a fosfolipase C- $\beta$ l (PLC- $\beta \mathrm{l})$ de maneira independente e se-

\section{perspectivas}

\author{
Oswaldo Luis Bracco \\ Márcia Jeha Kayath \\ José Gilberto H. Vieira
}

Eli Lilly do Brasil (OLB,MJK) e Disciplina de Endocrinologia, Universidade Federal de São Paulo (JGHV), São Paulo, SP. 
parada. Fragmentos de PTH sem os aminoácidos 1-34 da fração N-terminal, hPTH-(3-34), hPTH-(13-34) e hPTH-(8-84), ligam-se ao receptor ativando a PLC$\beta 1$ sem ativar a adenilciclase. Inversamente, fragmentos de PTH truncados na sua porção C-terminal podem ativar a adenilciclase, mas não a PLC- $\beta 1$. Assim o PTH (1-34) atua somente via adenilciclase (2).

O PTH aumenta a excreção renal de fósforo, aumenta a reabsorção renal de cálcio e acelera a conversão da 25-hidroxicolecalciferol em 1,25-dihidroxicolecalciferol e assim, indiretamente, aumenta a absorção intestinal de cálcio e fósforo. No tecido ósseo, o PTH atua nos osteoblastos promovendo a síntese e secreção de um ativador local de osteoclastos, aumentando a atividade osteoclástica. Esta ação aumenta a reabsorção óssea. Entretanto, há muito tempo, notou-se que o PTH poderia também exercer um efeito anabólico sobre o tecido ósseo, o que aumentaria a massa óssea (1). Há muito tempo, Reeve e cols. (3) descreveram que pacientes com osteoporose tratados com doses intermitentes de PTH (1-34) via subcutânea apresentavam aumento da massa de osso trabecular. Desde então, inúmeras investigações foram realizadas para avaliar a ação anabólica do PTH (1-34). A injeção de uma dose pequena de PTH nativo ou qualquer fragmento contendo os aminoácidos 1-34 (fragmento $\mathrm{N}$ terminal estimulante da adenilciclase) é capaz de estimular o crescimento cortical e a aumentar a espessura trabecular, sendo a ação anabólica de maior magnitude que a ação reabsortiva (2). A maneira de administração é crucial para a ação anabólica do $\mathrm{PTH}$, já que a infusão contínua da mesma dose do hormônio ou de grandes doses de um de seus fragmentos biologicamente ativos promove o predomínio da ação reabsortiva em relação à ação anabólica $(2,4,5)$. O PTH incrementa a massa óssea pelo aumento do número e da atividade dos osteoblastos (2). O PTH também reduz a apoptose dos osteoblastos (6). Assim, ocorre a deposição de camadas compactas de osso lamelar por ação dos osteoblastos, o que aumenta a resistência e a conectividade das trabéculas ósseas (2). Além disso, estudo pré-clínicos demonstraram que o PTH pode promover a reparação de fraturas, pelo aumento da massa óssea e do volume do calo ósseo em 75 e $99 \%$, respectivamente em relação ao controle, como observado 40 dias após fratura de tíbias em animais (2).

O PTH recombinante humano, PTH (1-34), foi desenvolvido como um agente terapêutico anabólico para o tratamento da osteoporose. Sua seqüência de aminoácidos é idêntica aos aminoácidos 1-34 da seqüência do PTH humano e sua afinidade ao receptor $\mathrm{PTH} / \mathrm{PTHrP}$ é similar à do $\mathrm{PTH}$ humano (7) (figura l).

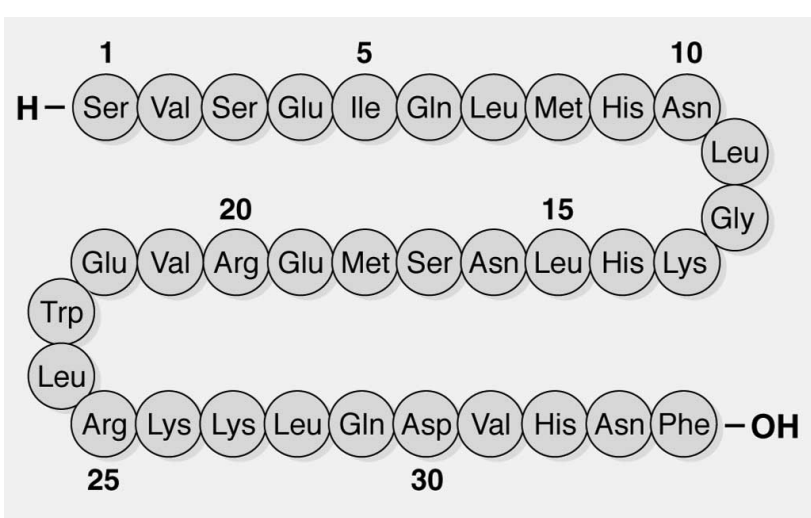

Figura 1. PTH (1-34) recombinante humano. A seqüência dos aminoácidos 1-34 é idêntica à do PTH endógeno.

Neste artigo de revisão, discutimos os artigos publicados na literatura e a eficácia e segurança do PTH (1-34) no tratamento da osteoporose.

\section{Estudos pré-clínicos}

O efeito do PTH (1-34) foi avaliado através de estudos em modelos animais. Macacas ooforectomizadas (8) receberam doses de PTH (1-34) de 0,1 ou $5 \mu \mathrm{g} / \mathrm{kg}$ administradas por até 18 meses ou por 12 meses seguidos de 6 meses sem tratamento. O tratamento foi bem tolerado e estimulou a formação de osso na vértebra, colo femural, tíbia proximal e crista ilíaca, resultando em aumento do volume de osso trabecular e da espessura cortical. A resistência óssea aumentou de maneira dose dependente, na coluna em $41 \%$ e colo femural em $23 \%$. Seis meses após a suspensão da medicação, persistia o aumento do volume ósseo e a maior resistência óssea. O efeito do PTH (1-34) no osso cortical foi melhor avaliado em coelhas ooforectomizadas que receberam 10 ou $40 \mu \mathrm{g} / \mathrm{kg} /$ dia de PTH (1-34) por 35,70 ou 140 dias. Observou-se aumento da massa óssea, aumento da área de secção transversal e da resistência femural $(9,10)$. Doses de PTH (1-34) de 8$40 \mu \mathrm{g} / \mathrm{kg} /$ dia via subcutânea, administradas a ratos (fêmeas normais, ooforectomizadas e machos normais) por 1 ano, promoveram aumento da massa óssea em até $154 \%$ na tíbia proximal e $72 \%$ na diáfise média femural e aumento da resistência óssea. Em ratas ooforectomizadas, o PTH (1-34) estimulou a aposição óssea nas superfícies trabecular, endosteal e periosteal, aumentando a resistência a fraturas nas vértebras, colo e diáfise femurais.

Os efeitos observados dependem da duração da exposição à droga. A avaliação farmacocinética do PTH (1-34) nestes estudos sugere que a resposta óssea ao PTH (1-34) (anabólica ou catabólica) é determinada primariamente pelo tempo de exposição por dia em 
que a concentração sérica de PTH (1-34) permanece acima da concentração basal do PTH endógeno. O PTH ou seus fragmentos amino-terminais promovem um efeito anabólico no tecido ósseo quando a administração é realizada por injeções diárias e promovem efeito predominantemente reabsortivo se administrados por infusão contínua que promova elevação persistente da concentração sérica de PTH (11).

\section{Farmacologia clínica}

Os estudos farmacológicos clínicos foram baseados nos principais estudos clínicos em homens e mulheres normais, assim como em pacientes com insuficiência renal leve a severa, hipertensão ou insuficiência cardíaca leve a moderada. Os dados de farmacocinética foram obtidos através de métodos tradicionais após injeção de 5-100 $\mu \mathrm{g} / \mathrm{kg}$ via subcutânea de PTH (1-34). Dados também foram obtidos a partir de estudo em mulheres na pós-menopausa com osteopenia ou osteoporose da pós-menopausa e em homens que participaram de um estudo fase II e 2 estudos fase III, respectivamente (12). Após uma injeção subcutânea, em média 95\% do PTH (1-34) estava biodisponível. A medicação apresentou absorção e eliminação rápidas. $\mathrm{O}$ pico da concentração sérica do (1-34) ocorreu 30 minutos após a injeção de uma dose de $20 \mu \mathrm{g}$ e decaiu para concentrações indetectáveis após 3 horas. A meia-vida na circulação sistêmica foi de 1 hora, em decorrência da aplicação via sub-cutânea, já que a meia-vida do PTH endógeno é bastante curta: 2-4 minutos. Não houve acúmulo do PTH (1-34) após várias doses diárias. A concentração sérica de PTH (1-34) excede rapidamente (minutos) o limite normal superior observado para o PTH endógeno, sendo indetectável 3-4 horas após a injeção sub-cutânea (tabela 1 ). Não há diferença relacionada à aplicação na parede abdominal ou coxa (12).

Não foram necessárias correções da dose de PTH (1-34) em pacientes com disfunção renal, pois não se observou alteração da depuração renal do PTH (1-34) em pacientes com insuficiência renal (12). A

Tabela 1. Propriedades farmacocinéticas da Teriparatida (PTH(1-34)rh).

\begin{tabular}{ll}
\hline Propriedade & Teriparatida \\
\hline Absorção & Rápida \\
Tempo para pico sérico & 30 minutos \\
Biodisponibilidade & $95 \%$ \\
Meia-vida & 60 minutos \\
Metabolismo & Fígado e rins \\
Eliminação & Rápida - em 3 horas \\
\hline
\end{tabular}

Arq Bras Endocrinol Metab vol $47 n^{\circ} 3$ Junho 2003 presença de insuficiência cardíaca não interferiu na farmacodinâmica do PTH (1-34) (12). O PTH (1-34) é metabolizado no fígado na célula de Kuppfer e não no hepatócito, assim a administração de PTH (1-34) pode ser indicada em pacientes com disfunção hepática (13). Os estudos farmacológicos clínicos demonstram que a administração de PTH (1-34) pode ser indicada para mulheres na pós-menopausa e homens independentemente da idade, peso corporal, tabagismo ou etilismo e do local escolhido para aplicação via subcutânea (12).

\section{Estudos clínicos}

Os estudos clínicos realizados com o PTH (1-34) avaliaram a eficácia da medicação na redução de fraturas vertebrais e extra-vertebrais, o efeito sobre a densidade mineral óssea (DMO) e nos marcadores do metabolismo ósseo em mulheres na pós-menopausa e em homens (12).

Neer e cols. avaliaram o efeito do PTH (1-34) em mulheres com osteoporose da pós-menopausa com uma fratura vertebral moderada ou duas fraturas não traumáticas ou com $\mathrm{DMO}<-1$ desvio-padrão da média de mulheres na pré-menopausa (14). Foram avaliadas 1637 mulheres ( 544 no grupo placebo, 541 no grupo dose $20 \mu \mathrm{g} / \mathrm{dia}, 552$ no grupo dose $40 \mu \mathrm{g} / \mathrm{dia})$. A duração média de tratamento de cada grupo foi de 18 meses. A DMO aumentou em média 9 e $13 \%$ com

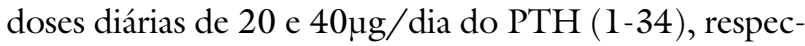
tivamente. O tratamento com PTH (1-34) 20 e $40 \mu \mathrm{g} /$ dia, respectivamente, em comparação com o placebo, reduziu significantemente o risco de uma ou mais novas fraturas vertebrais em 65 e 69\%; o risco de 2 ou mais novas fraturas vertebrais em 77 e $86 \%$ e o risco de uma fratura vertebral moderada ou severa em 90 e $78 \%$ (tabela 2). O número de fraturas vertebrais por paciente por ano foi 136 no grupo placebo, 49 no grupo $20 \mu \mathrm{g} /$ dia e 30 no grupo $40 \mu \mathrm{g} /$ dia. Uma fratura vertebral foi prevenida para cada 12 pacientes/ano de tratamento com a dose $20 \mu \mathrm{g} /$ dia e para cada 10 pacientes/ano com a dose $40 \mu \mathrm{g} / \mathrm{dia}$. O tratamento também reduziu significantemente o risco de fraturas não vertebrais por fragilidade em 53 e $54 \%$ nos grupos $20 \mu \mathrm{g} /$ dia e $40 \mu \mathrm{g} /$ dia, respectivamente. O efeito protetor tornou-se evidente após 9-12 meses de tratamento dependendo do sítio analisado.

Após o término do período de tratamento com PTH (1-34), 77\% das pacientes foram seguidas por mais 18 meses, com uma visita 6 meses após a suspensão do tratamento com PTH (1-34) e outra após 18 meses. Neste período foi permitido que as mulheres recebessem o tratamento de medicações anti-reabsorti- 
Tabela 2. Efeito da Teriparatida (PTH(1-34) $20 \mu \mathrm{g} / \mathrm{kg} / \mathrm{dia}$ ) na incidência e no risco das fraturas vertebrais em mulheres com osteoporose da pós menopausa.

\begin{tabular}{lccc}
\hline & \multicolumn{2}{c}{ Proporção de pacientes com fratura (\%) } \\
\cline { 2 - 4 } & $\begin{array}{c}\text { Teriparatida } \\
\mathrm{n}=444\end{array}$ & $\begin{array}{c}\text { Placebo } \\
\mathrm{n}=448\end{array}$ & $\begin{array}{c}\text { Redução } \\
\text { do risco } \\
\text { relativo } \\
(\%)\end{array}$ \\
\hline $\begin{array}{l}\text { Nova fratura }(\geq 1) \\
\text { Múltiplas fraturas }(\geq 2)\end{array}$ & 5,0 & 14,3 & 65 \\
$\begin{array}{l}\text { Fraturas moderadas } \\
\text { Ou severas }\end{array}$ & 0,9 & 4,9 & 77 \\
Fratura severa $(\geq 1)$ & 0,0 & 9,4 & 90 \\
\hline
\end{tabular}

vas. Não houve diferença estatística entre o número de pacientes que iniciaram tratamento com medicação anti-reabsortiva no grupo placebo e nos grupos PTH (1-34). Observou-se que as pacientes que haviam utilizado PTH (1-34) apresentavam menor risco de nova fratura vertebral, nova fratura vertebral moderada ou severa e de fraturas não vertebrais por fragilidade que as pacientes que estavam anteriormente no grupo placebo $(\mathrm{p}<0,05$ para todas as variáveis analisada) (12). O uso de PTH (1-34) reduziu a perda de altura das pacientes e foi associado à diminuição de dor lombar ou sua piora em relação ao placebo (14).

Lindsay e cols. (15) estudaram o efeito do PTH (1-34) $25 \mu \mathrm{g} /$ dia em 17 mulheres na pós-menopausa com osteoporose que vinham fazendo terapia de reposição hormonal (TRH), e comparou o resultado com as 17 pacientes submetidas somente à TRH. As pacientes em TRH não apresentaram variação percentual da DMO no quadril, coluna lombar e corpo total, enquanto as pacientes em uso de PTH (1-34) e TRH apresentaram aumento da DMO nos três sítios avaliados. Na coluna, o aumento da DMO já foi significantemente superior no sexto mês de terapêutica e manteve-se em elevação pelos 36 meses do estudo. Também no quadril, notou-se aumento significante da DMO após 30 meses de PTH (1-34) e TRH e no corpo inteiro após 24 meses. Estes dados demonstram a ação rápida e sustentada do PTH (1-34). Além disso, o aumento da DMO na coluna não ocorreu acompanhado de uma diminuição da DMO do fêmur, demonstrando que o aumento de osso em locais de osso predominantemente trabecular não são acompanhados de perda óssea de osso cortical (15), como observado no hiperparatireoidismo primário, que é caracterizado por perdas acentuadas de osso cortical (5). Os autores observaram que o aumento da DMO foi acompanhado de uma diminuição significante da incidência de fraturas vertebrais no grupo PTH (1-34) e TRH versus TRH isoladamente, apesar deste não ser um ponto de avaliação primária deste estudo (15).

Cosman e cols. (16) avaliaram 280 pacientes com osteoporose que vinham recebendo TRH ou terapia de reposição estrogênica (TRE) há pelo menos 1 ano. As pacientes foram divididas randomicamente em dois grupos: TRH/TRE somente e outro que manteve-se a TRH/TRE associando-se PTH (1-34) $25 \mu \mathrm{g} /$ dia via subcutânea diariamente. As pacientes foram observadas por 3 anos e a seguir por mais um ano de após a descontinuação do PTH (1-34). Observou-se aumento da DMO na coluna lombar, quadril e corpo inteiro, e diminuição significante do risco de fraturas vertebrais no grupo PTH (1-34) e TRH/TRE após 36 meses de terapêutica. Interessantemente, estes autores observaram que o incremento da massa óssea que ocorreu com o uso de PTH (1-34) pode ser mantido por até 2 anos após a descontinuação desta terapêutica e manutenção da TRH/TRE. Desta forma, observaram que o incremento da DMO nos diferentes locais estudados não seria transitório e sim prolongado.

Os bisfosfonatos suprimem intensamente a remodelação óssea. Por isso são freqüentemente utilizados para tratamento da osteoporose severa ou estabelecida. Por esse motivo, um grande número de investigações têm sido realizadas para avaliar a associação de PTH (1-34) ao tratamento com bisfosfonato ou para determinar se o uso prévio de bisfosfonato afeta a resposta ao PTH (1-34).

Ettinger e cols. (17) avaliaram a resposta ao PTH (1-34) de 33 pacientes que estiveram usando alendronato $10 \mathrm{mg} /$ dia por pelo menos 18 meses antes de iniciar imediatamente PTH (1-34)rh $20 \mu \mathrm{g} /$ dia para tratamento da osteoporose pósmenopausa. A média de tratamento com alendronato era de 27,8 $\pm 5,0$ meses \pm DP. Após um mês de terapêutica com PTH (1-34), observou-se elevação significante dos marcadores da formação óssea (osteocalcina, fosfatase alcalina óssea específica e PINP) e de reabsorção óssea (CTX e NTX). Os autores concluíram que existe uma resposta adequada ao tratamento com PTH (1-34) naquelas pacientes previamente tratadas com alendronato.

Ma e cols. (18) avaliaram ratas ooforectomizadas previamente tratadas com alendronato por 10 meses tratadas seqüencialmente com PTH SC por 2 meses. Os autores observaram um aumento de $113 \%$ na área trabecular.

Neer e cols. (19), assim como Finkelstein e cols. (20), observaram que a adição de PTH (1-34) a homens e mulheres previamente utilizando alendronato não 
reduz a resposta observada na variação da DMO quando comparada com o grupo utilizando cada droga separadamente.

Halladay e cols. (21) avaliaram a expressão gênica em células SaOS-2 para avaliar a ação do alendronato e do PTH (1-34) na formação óssea. O alendronato não estimulou a expressão gênica de Runx-2 e osteocalcina, entretanto a administração de alendronato (24hs) seguido de PTH (1-34) aumentou-a em 1,5-2 vezes.

Desta forma, não existem dados na literatura que contra-indiquem o uso de PTH (1-34) em pacientes previamente em uso de bisfosfonatos.

A osteoporose corticóide-induzida é a forma mais comum de osteoporose secundária (22). Lane e cols. (23) avaliaram o efeito do PTH (1-34) em 51 pacientes com osteoporose, no período pós-menopausa, em uso de TRH por mais de 1 ano e que estivessem em uso de prednisona ou equivalente por mais de 1 ano. As pacientes foram randomicamente divididas $\mathrm{em}$ dois grupos: PTH (1-34) e TRH $(\mathrm{n}=28)$ ou apenas TRH ( $n=23)$. O PTH (1-34) foi administrado via subcutânea em doses diárias de $25 \mu \mathrm{g}$. As pacientes do grupo PTH (1-34) e TRH apresentaram aumento significante da DMO da coluna lombar em relação às pacientes recebendo apenas TRH após 12 meses de tratamento. $\mathrm{O}$ incremento médio, avaliado por tomografia computadorizada quantitativa, observado no osso trabecular, foi de $35 \%$. No quadril, o incremento foi de $2 \%$ e não houve alteração no rádio distal. $\mathrm{O}$ PTH (1-34) foi capaz de estimular a formação óssea. As pacientes suspenderam o uso de PTH (1-34) após 12 meses. Observou-se que o incremento na massa óssea das pacientes em uso daquela medicação aumentou na coluna lombar nos 12 meses subseqüentes, nos quais as pacientes receberam apenas TRH e glicocorticóide. Portanto, o ganho de massa óssea promovido pela administração de PTH (1-34) foi mantido pelo período de observação de mais 12 meses (24).

A incidência média de fraturas anuais aumenta exponencialmente nos homens com o decorrer da idade, mas este incremento ocorre 5-10 anos depois do incremento exponencial observado nas mulheres (25). Aproximadamente $30 \%$ das fraturas do quadril incidem em homens (25). Kurland e cols. (26) avaliaram os efeitos de doses diárias de PTH (1-34) 400UI via subcutânea em homens com osteoporose idiopática com baixa remodelação óssea. Os homens foram aleatoriamente divididos em dois grupos $(\mathrm{n}=10$ em PTH (1-34) e $\mathrm{n}=13 \mathrm{em}$ placebo) e avaliados por 18 meses. O grupo em PTH (1-34) apresentou aumento linear da DMO na coluna lombar e colo do fêmur com incremento significante de $13,5 \pm 3,0 \%$ e $2,9 \pm 1,5 \%$ (média \pm desvio-padrão), respectivamente aos 18 meses, em comparação com o grupo placebo que não apresentou incremento. Todos os marcadores da remodelação óssea aumentaram no grupo tratado com PTH (1-34). O PTH (1-34) em doses baixas e intermitentes foi eficaz no tratamento da osteoporose idiopática masculina, pois aumentou a DMO após 18 meses de terapêutica. Recentemente, Orwoll e cols. (27) relataram que o PTH (1-34) reduziu o risco de fraturas vertebrais moderadas ou severas em $83 \%$ quando comparado ao placebo, em homens com osteoporose $(n=437)$ observados por 23 meses (11 meses de terapia mais 12 meses após a suspensão da medicação). Este resultado foi observado independentemente da concentração sérica de testosterona.

\section{Microarquitetura óssea}

A administração intermitente de PTH (1-34) promove um aumento rápido e prolongado da remodelação óssea, como demonstrado pela histomorfometria realizada em tecido ósseo obtido através de biópsia da crista ilíaca de 29 pacientes portadoras de osteoporose severa, em relação ao grupo controle (28). Houve incremento de osso trabecular e cortical, resultado da ação anabólica do PTH (1-34) nestes dois locais (28). Esta ação anabólica, tanto em osso cortical como trabecular, foi confirmada por outros autores, que demonstraram ainda que o PTH (1-34) apresenta efeito na microarquitetura óssea: aumento da espessura cortical e dados sugestivos de melhora da conectividade óssea (29-31).

\section{Segurança}

O programa clínico do PTH (1-34) constituiu-se de 25 estudos clínicos, com mais de 2800 homens e mulheres (12). Não se observou risco aumentado para qualquer neoplasia em pacientes que utilizaram o PTH (1-34) durante os estudos clínicos fase III ou após a suspensão da medicação (12). Macacas recebendo doses variáveis de PTH $(1-34)(20-40 \mu \mathrm{g} / \mathrm{kg})$ também não apresentaram osteossarcomas $(12,32)$. Ratas recebendo doses de $0,5,30$ ou $75 \mu$ de PTH (1-34) quase a totalidade da sua vida - doses maiores que a administrada por Neer e cols. (14) às mulheres na pós menopausa $(\sim 0,3 \mu \mathrm{g} / \mathrm{kg})$-, apresentaram osteossarcomas cuja incidência foi dose dependente $(12,32)$. A resposta exacerbada em ratas decorre de diferenças fundamentais entre a fisiologia do tecido ósseo humano e das ratas, cujo osso cresce durante toda a vida do animal sob estímulo do PTH (maior sensibili- 
dade ao PTH) e não apresenta remodelação óssea. Por isso, acredita-se que os achados de osteossarcomas em ratas não são transponíveis para humanos, sendo provavelmente relacionados à diferente fisiologia do osso desses animais e não à utilização de PTH (1-34) (32). Além disso, pacientes com Hiperparatireioidismo Primário não tem incidência aumentada de neoplasia, nem de osteossarcoma (33).

Não houve diferença significante entre número de mortes e hospitalização entre pacientes recebendo PTH (1-34) e placebo. Não se observou diferença entre os grupos quanto à incidência de doenças cardiovasculares, hipertensão, urolitíase e gota. Também não foi observado nenhum caso de osteossarcoma (14).

\section{Eventos adversos}

Não houve diferença estatisticamente significante entre a incidência de náusea, cefaléia e da descontinuação do tratamento entre os grupos PTH (1-34) $20 \mu \mathrm{g} /$ dia e placebo no estudo relatado por Neer e cols. (14). Calcemia ao menos uma vez elevada e cãimbras foram relatadas mais freqüentemente no

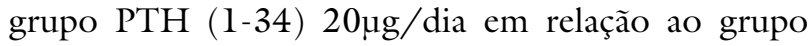
placebo, apesar da incidência ser baixa. A hipercalcemia foi dose dependente e predominantemente relacionada à dose de $40 \mu \mathrm{g} /$ dia. Hipercalcemia (duas mensurações realizadas com valores acima do normal) foi observada apenas em $3 \%$ das pacientes que rece-

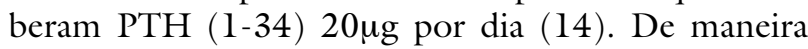
geral, observou-se que a hipercalcemia foi transitória e a concentração sérica de cálcio era normal antes da próxima injeção de PTH (1-34) (14).

\section{CONCLUSÃO}

O PTH (1-34) é uma medicação com ação anabólica no osso quando administrada via subcutânea na dose de $20 \mu \mathrm{g}$ diariamente. Aumenta a densidade mineral óssea da coluna lombar e do fềmur, aumenta a massa óssea e a conectividade óssea. Seu efeito ósseo reduz a incidência de fraturas vertebrais e extra-vertebrais em mulheres e homens. Sua ação no tecido ósseo é preservada após a suspensão da medicação. É uma medicação segura, com freqüência pequena de eventos adversos, os quais desaparecem após a descontinuação do tratamento. É um tratamento eficaz para o tratamento da osteoporose idiopática em homens e em mulheres com osteoporose pós-menopausa severa e fraturas ósseas induzidas por corticóide, constituindo-se na primeira terapia anabólica para tratamento destas situações clínicas.

\section{CONFLITO DE INTERESSES}

Os Drs. Oswaldo Luis Bracco e Márcia Jeha Kayath são médicos pesquisadores clínicos da Eli Lilly do Brasil.

\section{REFERÊNCIAS}

1. Macintyre I. The hormonal regulation of extracellular calcium. Brit Med Bull 1986;42:343-52.

2. Whitfield JF, Morley P, Willick GE. The bone-building action of the parathyroid hormone. Implications for the treatment of osteoporosis. Drug Aging 1999; 15:117-29.

3. Reeve J, Meunier PJ, Parsons JA, et al. Anabolic effect of human parathyroid hormone fragment on trabecular bone in involutional osteoporosis: a multicentre trial. $\mathbf{B r}$ Med J 1980;280:1340-4.

4. Tam CS, Heersche JNM, Murray TM, Parsons JA. Parathyroid hormone stimulates the bone apposition rate independently of its resorptive action: differential effects of intermittent and continuous administration. Endocrinology $1982 ; 110: 506-12$

5. Horwitz M, Stewart A, Greespan SL. Editorial: Sequential parathyroid hormone/alendronate therapy for osteoporosis - robbing Peter to pay Paul? J Clin Endocrinol Metab 2000;85:2127-8

6. Ali AA, O'Brien CAO, Weinstein RS, Roberson P, Manolagas $\mathrm{SC}$, Jilka RL. An inverse relationship between prevalence of osteoblast apoptosis and rate of bone formation with intermittent, but not sustained, elevation of PTH in mice. The ASBMR 23rd Annual Meeting, 2001 (abst. \#1 169).

7. Pliam NB, Nyiredy KO, Arnaud CD. Parathyroid hormone receptors in avian bone cells. Proc Natl Acad Sci USA 1982;79:2061-93.

8. Burr DB, Hirano T, Turner $\mathrm{CH}$, Hotchkiss C, Brommage R, Hock JM. Intermittently administered human parathyroid hormone (1-34) treatment increases intracortical bone turnover and porosity without reducing bone strength in the humerus of ovariectomized cynomolgus monkeys. J Bone Miner Res 2001;16:157-65.

9. Hirano T, Burr DB, Turner CH, Sato M, Cain RL, Hock JM. Anabolic effects of human biosynthetic parathyroid hormone fragment (1-34), LY333334, on remodeling and mechanical properties of cortical bone in rabbits. J Bone Miner Res 1999; 14:536-45.

10. Hirano T, Burr DB, Cain RL, Hock JM. Changes in geometry and cortical porosity in adult, ovary-intact rabbits after 5 months treatment with LY333334 (hPTH 1-34). Calcif Tissue Int 2000;66:456-60.

11. Tam CS, Heersche JN, Murray TM, Parsons JA. Parathyroid hormone stimulates the bone apposition rate independently of its resorptive action: differential effects of intermittent and continuous administration. Endocrinology $1982 ; 110: 506-12$.

12. PTH (1-34) Injection NDA 21-318. Endocrinologic and metabolic drugs advisory committee briefing document. Vol. 1. June 15th, 2001. http://www.fda.gov/ohrms/dockets/ac/01/briefing/3761b2_01_lilly.pdf 
13. Daugaard H.Peripheral metabolism of parathyroid hormone. Studies with isolated perfused kidneys and livers from normal and uremic rats. Dan Med Bull 1996;43:203-15.

14. Neer RM, Arnaud CD, Zanchetta JR, Prince R, Gaich GA Regisnter J-Y, et al. Effect of parathyroid hormone (1-34) on fractures and bone mineral density in postmenopausal women with osteoporosis. N Engl J Med 2001;344:1434-41

15. Lindsay R, Nieves J, Formica C, et al. Randomized controlled study of effect of parathyroid hormone on vertebral-bone mass and fracture incidence among postmenopausal women on oestrogen with osteoporosis. Lancet 1997;23:550-5.

16. Cosman F, Nieves J, Woelfert L, et al. Parathyroid hormone added to established hormone therapy: effects on vertebral fracture and maintenance of bone mass after parathyroid hormone withdrawn. J Bone Miner Res 2001;16:925-31.

17. Ettinger B, San Martin JA, Crans G, Pavo I. Early response of bone turnover markers and bone mineral density to teriparatide (recombinant human Parathyroid Hormone (1-34)) in postmenopausal women previously treated with an antiresorptive drug. J Bone Min Res 2002;17(Suppl 1):S157.

18. Ma YL, Zeng $Q Q$, Schmidt A, Hoover JL, Cole HW, Bryant $\mathrm{HU}$, et al. Teriparatide (rhPTH(1-34)) skeletal efficacy is not affected by longterm pretreatment with alendronate, estrogen or raloxifene in ovariectomized rats. $\mathbf{J}$ Bone Miner Research 2002;17(Suppl 1):S167.

19. Neer R, Hayes A, Rao A, Finkelstein J. Effects of Parathyroid Hormone, alendronate or both on bone density in osteoporotic postmenopausal women. J Bone Miner Research 2002;17(Suppl 1):S135.

20. Filkelstein JS, Hayes, Rao A, Neer RM. Effects of Parathyroid Hormone, alendronate or both on bone density in osteoporotic men. J Bone Miner Research 2002; 17(Suppl 1):S127.

21. Halladay DL, Martin TJ, Onyia JE. Osteogenic effects of PTH in osteoblast-like cells are enhanced by alendronate pre-treatment. J Bone Miner Research 2002; 17(Suppl 1):S273

22. Lukert BP, Raisz LG. Glucocorticoid-osteoporosis: pathogenesis and management. Ann Inter Med 1990; 1 12:352-64.

23. Lane NE, Sanchez S, Modin GW, Genant HK, Pierini E, Arnaud CD. Parathyroid hormone treatment can reverse corticosteroid-induced osteoporosis. Results of a randomized controlled clinical trial. J Clin Invest $1998 ; 102: 1627-963$

24. Lane NE, Sanchez S, Modin GW, Genant HK, Pierini E, Arnaud $\mathrm{CD}$. Bone mass continues to increase at the hip after parathyroid hormone treatment is discontinued in glucocorticoid-induced osteoporosis: results of a randomized controlled clinical trial. J Bone Miner Res 2000; 15:944-51.
25. Favus MJ. Primer on the metabolic bone diseases and disorders of mineral metabolism. Philadelphia: Lippincott-Raven, 1999.

26. Kurland ES, Cosman F, McMahon DJ, Rosen CJ, Lindsay R, Bilezikian JP. Parathyroid hormone as a therapy for idiopathic osteoporosis in men: effects on bone mineral density and bone markers. J Clin Endocrinol Metab 2000;85:3069-76.

27. Orwoll E, Scheele WH, Clancy AD, et al. Recombinant human parathyroid hormone (1-34) therapy reduces the incidence of moderate/severe vertebral fractures in men with low bone density. The ASBMR 23 ${ }^{\text {rd }}$ Annual Meeting, 2001 (abst. \#1 104).

28. Hodsman AB, Kisiel M, Adachi JD, Fraher LJ, Watson PH. Histomorfometric Evidence for increased bone turnover without change in cortical thickness or porosity after 2 years of cyclic hPTH(1-34) therapy in woman with severe osteoporosis. Bone 2000;27:311-8.

29. Dempster DW, Cosman F, Kurland ES, et al. Effects of daily treatment with parathyroid hormone on bone micro architecture and turnover in patients with osteoporosis: a paired biopsy study. J Bone Miner Res 2001:16:1846-53.

30. Seeman E, Delmas PD. Reconstructing the skeleton with intermittent parathyroid hormone. Trends Endocrinol 2001;12:281-3

31. Eriksen EF, Wang O, Genant HK, et al. Two- and 3-dimensional analysis of cancellous and cortical bone in a multicenter, double-blind, randomized and placebo-controlled study of teriparatide. American College of Rheumatology, 2002 (oral presentation).

32. Tashjian AH, Chabner BA. Commentary on clinical safety of recombinant human parathyroid hormone 1-34 in the treatment of osteoporosis in men and postmenopausal women. J Bone Miner Res 2002;17:1151-61.

33. Smith J, Huvos AG, Chapman M, Rabbs C, Spiro RH Hyperparathyroidism associated with sarcoma of bone. Skeletal Radiol 1997;26:107-12.

\section{Endereço para correspondência:}

Oswaldo Luis Bracco

Av. Morumbi 8264

04703-002 São Paulo, SP

e.mail: bracco_oswaldo@lilly.com 